\title{
Efecto de suplementos de zinc en la incidencia de caries y en la concentración de Inmunoglobulinas A salivales en niños escolares de talla baja
}

\author{
Janeth Parra ${ }^{1}$, Diana Astudillo ${ }^{2}$, Pablo Parra ${ }^{3}$, Fernando Sempértegui ${ }^{4}$ \\ ${ }^{1}$ Facultad de Odontología, Universidad de Cuenca, Cuenca, Ecuador. \\ ${ }^{2}$ Facultad de Ciencias Químicas, Universidad de Cuenca, Cuenca, Ecuador. \\ ${ }^{3}$ Médico Internista-Neumólogo, Hospital José Carrasco Arteaga, Cuenca, Ecuador. \\ ${ }^{4}$ Facultad de Ciencias Médicas, Universidad Central del Ecuador, Quito, Ecuador. \\ Autor de correspondencia: judith.parra@ucuenca.edu.ec \\ Fecha de recepción: 30 de abril 2013 - Fecha de aceptación: 17 de junio 2013
}

\begin{abstract}
RESUMEN
La población de niños en el Ecuador presenta una alta prevalencia de deficiencia crónica de zinc, asociada a una deficiencia de la inmunidad celular y humoral y un incremento elevado de caries dental. Esta investigación presenta el efecto de la ingesta de $10 \mathrm{mg}$ de sulfato de zinc versus el grupo control que recibe una solución con una sustancia inactiva durante 42 semanas y se observa el cambio detectado en relación al peso de los niños, la talla, la concentración de Inmunoglobulina A (IgA) en saliva, la concentración plasmática de zinc, y los índices de Klein y Palmer (CPOD y CPOS). En este estudio se consideró a la caries incipiente dentro del componente caries. Información paralela acerca de las condiciones de higiene y de vida fueron recolectadas utilizando cuestionarios estructurados. El análisis estadístico de los datos recolectados reveló que el CPOS es menor y que la concentración final plasmática de zinc es más alta en el grupo experimental de niños quienes recibieron el suplemento diario de zinc $\left(17,30 \pm 9,55\right.$ vs. $20,03 \pm 10,74 ; \mathrm{p}=0,032 ; 123 \pm 24,29 \mu \mathrm{g} \mathrm{dl}^{-1}$ vs. $105,57 \pm 15,75 \mu \mathrm{g} \mathrm{dl}^{-1}$; $\mathrm{p}<0,001)$. El análisis de regresión lineal múltiple mostró que los suplementos de zinc están asociados con CPOS reducido (beta $=-3,9 ; p=0,033$ ) y un CPOD reducido (beta $=-1,33 ; p=0,05$ ) y que el zinc basal se asocia con una concentración final más baja de IgA en saliva (beta $=-0,043 ; p=0,011$ ). El cepillado con pasta dental se asoció con reducción del CPOS (beta $=-7,14 ; \mathrm{p}=0,033$ ). El enjuague con agua potable después del cepillado se asoció con reducción de CPOD (beta $=-1,42 ; p=0,035)$. El estudio reveló que suplementos de zinc y la higiene oral influyen positivamente la salud bucal, de la misma manera que producen un mejoramiento de la inmunidad de la mucosa oral, los cuales al ser aplicados van a reducir la magnitud de este problema de salud pública.
\end{abstract}

Palabras clave: CPOD, CPOS, zinc, IgA salival, higiene oral.

\begin{abstract}
Young children in Ecuador show high prevalence of chronic zinc deficiency, associated with a decline in cellular and humoral immunity, and increased risk of tooth decay. The research reported in this paper presents the effect of the daily consumption of $10 \mathrm{mg}$ zinc sulfate versus the control group receiving a solution with an inactive substance during 42 weeks, on respectively the children's weight, height, the immunoglobulin A ( $\operatorname{Ig} \mathrm{A})$ concentration in saliva, the plasma concentrations of zinc, and the indices of Klein and Palmer (DMFT and DMFS). In the survey special attention was given to the detection of early stage tooth decay, also called incipient decay. Parallel, information about the children's hygiene and living condition was collected using structured questionnaires. Statistical analysis of the collected data revealed that DMFS is less and the final concentration of plasma zinc higher in the experimental group of children receiving daily a zinc supplement (17,30 $\pm 9,55 \mathrm{vs.} 20,03$ $\pm 10,74 ; \mathrm{p}=0,032 ; 123 \pm 24,29 \mu \mathrm{g} \mathrm{dl}^{-1}$ vs. $\left.105,57 \pm 15,75 \mu \mathrm{g} \mathrm{dl}{ }^{-1} ; \mathrm{p}<0,001\right)$. Multiple linear
\end{abstract}


regression showed that zinc supplements are associated with reduced DMFS (beta $=-3,9 ; p=0,033$ ) and DMFT (beta $=-1,33 ; \mathrm{p}=0,05$ ), and that the basal state of zinc allies with a lower final concentration of IgA in saliva (beta $=-0,043 ; p=0,011$ ). More intensive toothbrush with the use of toothpaste was associated with a reduction of DMFS (beta $=-7,14 ; p=0,033$ ). Rinsing the mouth with potable water resulted in a decline of DMFT (beta $=-1,42 ; p=0,035$ ). The study revealed that zinc supplements and oral hygiene positively affect oral health, likely through an improvement of oral mucosal immunity, which when applied will reduce the magnitude of this public health problem.

Keywords: DMFT, DMFS, zinc, IgA salival, oral hygiene.

\section{INTRODUCCIÓN}

Según la Organización Mundial de la Salud (OMS/WHO), a la caries dental se la puede definir como proceso patológico, localizado, de origen externo, que se inicia tras la erupción dentaria y que determina un reblandecimiento del tejido duro del diente, evolucionando hacia la formación de una cavidad. Si no se atiende oportunamente, afecta la salud general y la calidad de vida de los individuos de todas las edades (WHO, 1987). Hoy se la considera como un proceso dinámico con períodos alternados de desmineralización y remineralización. En los países en desarrollo las prácticas de salud bucal están dirigidas al tratamiento de patologías más que a su prevención, manteniéndose en forma dominante una odontología curativa.

La infección oral persistente con afectación de las estructuras dentarias, de alta prevalencia en el país (Ministerio de Salud Pública del Ecuador, 1997), se asocia a defectos de la masticación (Peres y col., 2000) que menoscaban la buena digestión. Además, la caries dental se correlaciona con una deficiencia en el desarrollo psíquico de los niños (Struska y Mielnik-Blaszczak, 2003). Al mismo tiempo, esta deficiencia podría afectar el sistema inmune de la boca y favorecer la infección de la cavidad oral con daño de las piezas dentarias. La caries es un problema de alta prevalencia en los niños, que afecta la nutrición y el crecimiento.

El zinc es un mineral crítico en el crecimiento longitudinal de modo que el retraso de talla se considera un marcador de deficiencia de este mineral. Los países Andinos han sido considerados como una de las regiones del mundo con la más alta prevalencia de deficiencia crónica de zinc, lo cual se debe a dietas insuficientes y al consumo simultáneo de cereales no refinados con alto contenido de fitatos que interfieren la absorción de este mineral (IZiNCG, 2004).

Puesto que el zinc está involucrado también en la respuesta inmune, su deficiencia podría favorecer al desarrollo de la caries dental. La deficiencia de zinc afecta aproximadamente al $40 \%$ de los niños ecuatorianos (Freire, 1988) y la deficiencia de talla al 26\% en población urbana y al $40 \%$ en población rural de la Sierra (Larrea y col., 2001). Se conoce que el zinc está implicado en el crecimiento longitudinal por su alta concentración en los huesos y en el cartílago de crecimiento (Zhou y col., 1993). Se ha encontrado una estable y significativa correlación entre retraso de talla e inadecuada ingesta de zinc en diversas poblaciones del mundo (IZiNCG, 2004). Por eso, es ampliamente aceptado que el retraso de talla es un buen marcador de deficiencia de zinc en niños (IZiNCG, 2004). Recientemente, nosotros encontramos deficiencia de zinc plasmático (punto de corte $65 \mu \mathrm{g} \mathrm{dl}^{-1}$ ) en $31.8 \%$ de una muestra de niños de zonas semi-rurales de la costa y de zonas rurales de la sierra (Wuehler et al, 2008). Nuestros estudios (Sempértegui y col.,1996) y otros (Shankar y Prasad,1998; Fraker y col., 2000) han mostrado que el zinc mejora la inmunidad, especialmente la inmunidad celular. Varios trabajos evidencian que suplementos de zinc disminuyen la frecuencia de diarrea (Sazawal y col., 1997) y neumonía en niños (Bhutta y col., 1999). Se ha establecido firmemente que niños con retraso de talla recuperan su ritmo de crecimiento con suplementos de zinc (Brown y col., 2002).

Diversas evidencias muestran que el zinc participa en la maduración de los linfocitos $\mathrm{T}$ en el timo, linfocitos que son indispensables para la inducción de células plasmáticas productoras de anticuerpos (Beck y col., 1997; Prasad y col., 1988). Hay estudios que asocian el incremento de IgA salival con mejoramiento de la inmunidad oral contra los patógenos que causan caries (Lee y col., 
2004; Tar y col., 1999). Estudios experimentales en roedores demuestran que aumenta la susceptibilidad a la caries dental cuando hay deficiencia de Zinc (Cerklewski,1981; Johnson y col., 1984). Por tanto, nosotros conjeturamos que los suplementos de zinc administrados a niños con deficiencia de este mineral, deficiencia que estaría sugerida por la talla baja, tendrían un efecto protector contra las caries y podrían mejorar la inmunidad de la cavidad oral, en particular la concentración de IgA.

\section{MATERIALES Y MÉTODOS}

\subsection{Diseño experimental}

Se realizó un estudio clínico-experimental, doble ciego, controlado con placebo. Se enrolaron 297 niños de 6 a 12 años de edad, de seis escuelas urbano marginales de la ciudad de Cuenca, que mostraron retraso de talla para la edad $(<10$ percentil de las curvas del National Center Health Statistics de los Estados Unidos de América). Al ingreso, de cada niño se obtuvo información sobre hábitos de higiene dental, así como los servicios de agua y eliminación de excretas de la vivienda. Al inicio y al final de la suplementación se midió el peso y la talla, se estableció la concentración de zinc en plasma sanguíneo, el Índice de Caries (CPOD), el Índice de Superficies Cariadas (CPOS) y la concentración de IgA. Mediante procedimiento aleatorio se establecieron 2 grupos: un grupo suplementado (GZ) y grupo placebo (GP). El grupo GZ recibió $10 \mathrm{mg} \mathrm{día}^{-1}$ de zinc como sulfato y el GP recibió una solución de características organolépticas similares pero sin la sustancia activa. El régimen duró 42 semanas.

En poblaciones similares, se ha establecido que el índice de piezas cariadas, perdidas y obturadas (CPOD) es $>6$ en el 75\% de los niños afectados (Nicolau y col., 2005). Se estimó un efecto del zinc para reducir el CPOD a 3,15 $\pm 1,7$ (50\% de reducción). Por lo tanto, el tamaño de cada grupo, con un nivel de confianza del $95 \%$ y una precisión de 0,5 fue 180 . Se estimó una pérdida de $5 \%$, de modo que dentro de las estimaciones establecidas se requirieron 200 niños en cada grupo. (Smith y Morrow, 1991). Niños escolares de 6 a 12 años de las escuelas urbano marginales, que se encontraban en buen estado de salud general, con retraso de talla para la edad, que no estaban sometidos a ningún tratamiento farmacológico ni sometidos a atención odontológica. Se obtuvo el consentimiento informado de los padres de los niños y el asentimiento verbal de los niños.

\subsection{Procedimientos técnicos}

Los indices $^{1}$ de Klein y Palmer (CPOD y CPOS) y de Gruebbel (ceod y ceos) fueron establecidos mediante inspección clínica. Brevemente antes del examen se efectuó una profilaxis buco-dental, se limpiaron y se secaron bien las superficies respectivas (libres, proximales y oclusales). Entonces, se efectuó observación y exploración mediante el uso del espejo bucal, explorador con punta redondeada e hilo dental, de la siguiente manera. En las superficies libres y proximales para el diagnóstico de caries primaria (incipiente) se identificó la presencia de mancha blanca con bordes netos, aspecto opaco o retención del explorador. A nivel de superficies oclusales se buscó presencia de fisuras con mancha blanca. El diagnóstico de caries de segundo, tercer y cuarto grados se estableció por la presencia de cavidades abiertas.

La determinación de IgA en saliva se realizó mediante la técnica ELISA (Hau y col., 2001) en el laboratorio de la Facultad de Química de la Universidad de Cuenca. Brevemente: se estimuló la secreción de saliva mediante masticación de una cinta de parafina (Proctor y Carpenter, 2001), se la

\footnotetext{
${ }^{1}$ Índice CPOD: Se obtuvo de la suma de dientes permanentes cariados perdidos y obturados. Índice ceod: Se obtuvo de la suma de dientes primarios cariados, con indicación de extracción indicada "e" y obturados. Para la dentición mixta se sumó CPOD + ceod.

Índice CPOS: Se obtuvo de la suma de las superficies de los dientes permanentes cariados, perdidos y obturados. Índice ceos: Se obtuvo de la suma de las superficies de dientes primarios cariados, con indicación de extracción indicada "e" y obturados.

Para la dentición mixta se sumó CPOS + ceos (PRECONC, 1992).
} 
recogió en recipiente plástico estéril, se mantuvo en termo frío a $4^{\circ} \mathrm{C}$ y se transportó al laboratorio dentro 1-2 horas. En el laboratorio la muestra fue centrifugada para descartar las células y el sobrenadante se guardó a $-20^{\circ} \mathrm{C}$ hasta el análisis. La identificación de anticuerpos se realizó con un kit de la empresa Bethyl (Bethyl Laboratories Inc., Montgomery TX). Se siguieron estrictamente las recomendaciones del fabricante. La absorbancia se leyó en un lector de MicroELISA con filtro de 450 $\mathrm{nm}$ (Universal Microplate Reader, Texas Instruments). Cada muestra se procesó en duplicado y se dispuso de estándares proporcionados por el mismo kit. Del promedio de las dos mediciones se restó la absorbancia del blanco. El valor obtenido se llevó a intersección con la curva estándar y se calculó la concentración final en $\mathrm{mg} \mathrm{dl}^{-1}$ de IgA. Muestras basales y finales se analizaron conjuntamente siguiendo un diseño ciego para evitar sesgos.

La talla se midió mediante procedimiento estandarizado (doble medición) y se registró en centímetros y décimas. El programa EpiInfo, NUTSTAT versión CDC, 2004, sirvió para establecer el "score" Z de talla/edad (HAZ) según los estándares de los Centros de Estadística de los Estados Unidos (National Center Health Statistics-NCHS). El peso se midió mediante procedimiento estandarizado en una balanza. Se calculó también el "score" $Z$ de peso/edad (WAZ) como se describió para HAZ.

El jarabe de sulfato de zinc y el placebo fueron preparados en la Facultad de Química de la Universidad Central, Quito, que sigue los procedimientos de buenas prácticas de manufactura (GMP). La determinación de zinc en plasma sanguíneo se realizó en los laboratorios de la misma Facultad de Química de la Universidad Central, en Quito, mediante espectrofotometría de absorción atómica como está descrito (Sempértegui y col., 1996). La sangre se obtuvo por punción venosa en la mañana con el niño en ayunas de 12 horas.

\subsection{Randomización y administración del suplemento}

La asignación aleatoria de los niños a los grupos zinc (GZ) y placebo (GP) se realizó mediante distribución de las cuatro series de frascos de jarabe que fueron entregadas por el productor con códigos de letras: $\mathrm{R}$ par, $\mathrm{R}$ impar, $\mathrm{C}$ par, $\mathrm{C}$ impar. Las escuelas fueron sorteadas para una de las series de frascos. El Comité de Ética guardó el sobre sellado proporcionado por el fabricante que contenía los códigos de la sustancia activa (zinc) o placebo. El suplemento (zinc o placebo), de similar apariencia y sabor, contenido en los frascos codificados, fue administrado a los niños cada día. En los días de escuela un maestro (a) entrenado (a) fue responsable de la administración del jarabe. Los investigadores visitaron las escuelas una vez por semana para verificar el cumplimiento del régimen de suplementación y para identificar cualquier efecto indeseable.

\subsection{Análisis estadístico}

$\mathrm{Al}$ inicio y al final de la suplementación se calculó el promedio y la desviación estándar (DE) de la edad, del CPOD, del CPOS, de la concentración de IgA, concentración de zinc en plasma, del score Z para peso (WAZ) y del score Z para talla (HAZ). Las diferencias entre los grupos suplementado y placebo se examinaron mediante la prueba Student-t-test. También al inicio se estableció el porcentaje de niños según género, hábitos de higiene oral y condiciones de vida. Las diferencias entre los grupos suplementado y placebo se examinaron mediante la prueba de Chi-cuadrado.

Se diseñaron Modelos de Regresión Lineal Múltiple para: 1) examinar la asociación de los suplementos (zinc/placebo) con los índices finales CPOD y CPOS. Se controlaron las siguientes variables basales: HAZ, WAZ, la concentración de IgA, la concentración de zinc, las prácticas de higiene oral y el género; 2) examinar la asociación de los suplementos (zinc/placebo), con HAZ final. Se controlaron las siguientes variables basales: WAZ, la concentración de IgA, la concentración de zinc, las prácticas de higiene oral, el género, CPOD y CPOS, y, 3) examinar la asociación de los suplementos (zinc/placebo), con la concentración final de IgA. Se controlaron las siguientes variables basales: HAZ, WAZ, la concentración de zinc, las prácticas de higiene oral, el género, CPOD y CPOS. El límite de significación aceptado fue $\mathrm{p}<0,05$. Todos los análisis se realizaron con el programa SPSS, versión 11.5. 


\section{RESULTADOS}

\subsection{Datos basales globales}

Se midió el peso y la talla a 1476 niños escolares entre Diciembre del año 2005 y Febrero del año 2006. De estos, 997 niños tuvieron la talla bajo el percentil 10 del estándar del NCHS. A los padres de estos niños se solicitó firmar el consentimiento informado y se obtuvieron 400 consentimientos. $\mathrm{Al}$ retirarse una escuela por cambiar de horario diurno al de la tarde quedaron 297 niños enrolados en el estudio. Mediante asignación al azar, 147 niños quedaron en el grupo suplementado (GZ) y 150 en el grupo placebo (GP). Las mediciones basales se realizaron entre Julio del 2006 y Enero del 2007 porque a más de la evaluación dental, se efectuaron inactivaciones de caries, extracciones y aplicación de flúor. En este lapso, 37 niños se retiraron antes de que se inicie la suplementación y de que se completen todas las mediciones basales, por las siguientes causas: 15 porque sus padres retiraron el consentimiento, 20 por cambio de escuela, y 2 porque se cambiaron de domicilio. Se inició y terminó el estudio con 260 niños distribuidos en las diferentes escuelas. Al grupo suplementado pertenecieron 123 niños y al grupo placebo 137 (Fig. 1). La suplementación se extendió desde Febrero del 2007 hasta Enero del 2008, con interrupción desde el 15 de Julio hasta el 10 septiembre del 2007, debido al período de vacaciones anuales.

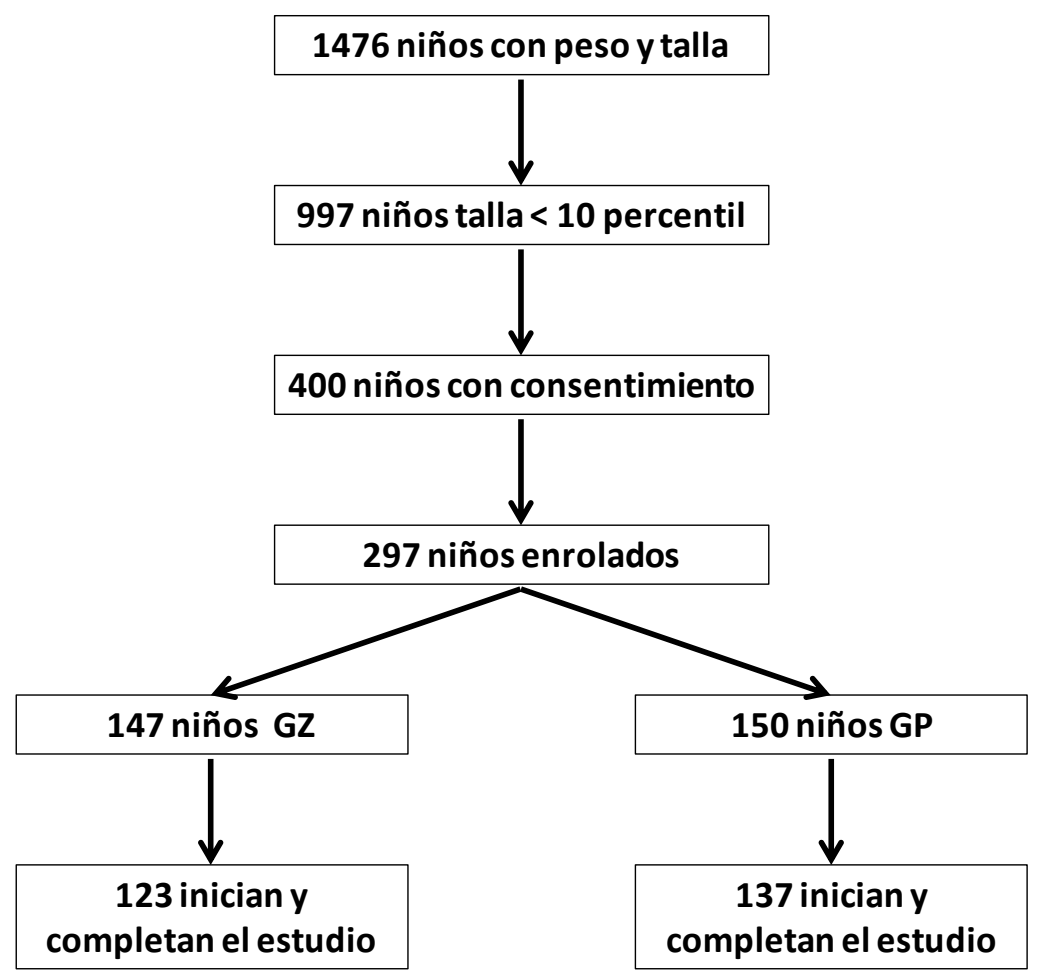

Figura 1. Pre-selección, selección y enrolamiento de los niños en el estudio.

\subsection{Comparaciones basales entre los grupos suplementado (GZ) y placebo (GP)}

Antes de iniciar la suplementación los dos grupos, suplementado (GZ) y placebo (GP), fueron similares en indicadores antropométricos, edad, CPOD, CPOS, concentración plasmática de zinc e Inmunoglobulina A en saliva (IgA). Hubo más niñas en el GP (Tabla 1). También fueron similares los indicadores de higiene oral y de condiciones de vida, con excepción del acceso a la red municipal de agua potable que fue mayor en el GP (Tabla 2). 
Tabla 1. Características basales de los grupos suplementado y placebo.

\begin{tabular}{llll}
\hline Características & \multicolumn{1}{c}{$\begin{array}{c}\text { Placebo } \\
\mathrm{n}=137 \\
\text { Media } \pm \text { DE }\end{array}$} & $\begin{array}{c}\text { Zinc } \\
\mathrm{n}=123\end{array}$ & $\mathrm{p}$ \\
& 78,8 & 48,7 & $<0,001$ \\
Sexo femenino (\%) & $104,22 \pm 20,55$ & $107,32 \pm 20,30$ & \\
Edad (meses) & $25,17 \pm 5,51$ & $25,69 \pm 5,20$ & \\
Peso (Kg) & $-0,80 \pm 0,77$ & $-0,83 \pm 0,74$ & \\
WAZ (DE) & $119,65 \pm 8,78$ & $121,98 \pm 9,02$ & \\
Talla (cm) & $-1,92 \pm 0,69$ & $-1,73 \pm 0,64$ & \\
HAZ (DE) & $17,37 \pm 1,64$ & $17,09 \pm 1,67$ & \\
BMI & $104,10 \pm 15,55$ & $106,82 \pm 21,84$ & \\
Zinc (ug dl $\left.{ }^{-1}\right)$ & $8,65 \pm 6,59$ & $7,29 \pm 5,54$ & \\
IgA (mg dl $\left.{ }^{-1}\right)$ & $8,95 \pm 3,46$ & $9,19 \pm 3,90$ & \\
CPOD & $16,15 \pm 8,27$ & $17,60 \pm 10,26$ & \\
CPOS & & & \\
\hline
\end{tabular}

Tabla2. Características basales de higiene oral y condiciones de vida familiar de los grupos suplementado y placebo.

\begin{tabular}{lcc}
\hline Características & $\begin{array}{c}\text { Placebo } \\
\mathrm{n}=137 \\
\%\end{array}$ & $\begin{array}{c}\text { Zinc } \\
\mathrm{n}=123 \\
\%\end{array}$ \\
\hline Cepillado dientes en la mañana & 54,01 & 50,41 \\
Cepillado dientes antes de acostarse & 51,82 & 48,8 \\
\hdashline Material para cepillado & 76,64 & 68,29 \\
Pasta dental & 0,73 & \\
Jabón & 1,46 & 1,63 \\
Ninguno & 71,53 & 41,46 \\
\hline Agua de consumo & 1,46 & 0,81 \\
Red Municipal & 1,46 & 0,81 \\
Pozo & 5,84 & 1,63 \\
Tanquero & - & 2,44 \\
Vertiente & - & 28,46 \\
Ninguna & 0,73 & - \\
Entubada & & \\
Río & 63,50 & 58,54 \\
Eliminación de excretas & 8,03 & 2,44 \\
Servicio Higiénico & 3,65 & 7,32 \\
Letrina & 5,11 & 6,50 \\
Pozo ciego & & \\
Ninguno & &
\end{tabular}

\subsection{Comparaciones finales entre los grupos suplementado (GZ) y placebo (GP)}

Al finalizar la suplementación el grupo que recibió zinc (GZ) tuvo un menor Índice CPOS, que el grupo que recibió placebo $(17,30 \pm 9,55$ vs. $20,03 \pm 10,74 ; \mathrm{p}=0,032)$. También el grupo suplementado tuvo mayor concentración de zinc en el plasma que el grupo placebo $(123,03 \pm 24,29$ vs. $105,57 \pm 15,75 \mu \mathrm{g} \mathrm{dl}^{-1} ; \mathrm{p}<0,001$ ) (Tabla 3 ). 
Tabla 3. Características post-suplementación en los grupos suplementado y placebo.

\begin{tabular}{llll}
\hline Características & \multicolumn{1}{c}{$\begin{array}{c}\text { Placebo } \\
\mathrm{n}=137 \\
\text { Media } \pm \mathrm{DE}\end{array}$} & $\begin{array}{c}\text { Zinc } \\
\mathrm{n}=123 \\
\text { Media } \pm \mathrm{DE}\end{array}$ & $\mathrm{p}$ \\
\hline Sexo femenino (\%) & 78,8 & 48,7 & $<0,001$ \\
Edad (meses) & $116,22 \pm 20,55$ & $119,32 \pm 20,30$ & \\
Peso $(\mathrm{Kg})$ & $28,74 \pm 6,65$ & $29,80 \pm 10,55$ & \\
WAZ $(\mathrm{DE})$ & $-0,70 \pm 0,94$ & $-0,72 \pm 0,92$ & \\
Talla $(\mathrm{cm})$ & $125,60 \pm 8,69$ & $127,12 \pm 8,39$ & \\
HAZ $(\mathrm{DE})$ & $-1,79 \pm 0,73$ & $-1,72 \pm 0,64$ & \\
BMI & $17,98 \pm 2,13$ & $18,33 \pm 6,47$ & \\
Zinc $\left(\mu \mathrm{g} \mathrm{dl}^{-1}\right)$ & $105,57 \pm 15,75$ & $123,03 \pm 24,29$ & $<0,001$ \\
IgA $\left(\mathrm{mg} \mathrm{dl}^{-1}\right)$ & $7,83 \pm 6,17$ & $8,49 \pm 5,84$ & \\
CPOD & $10,76 \pm 4,09$ & $9,19 \pm 3,90$ & \\
CPOS & $20,03 \pm 10,74$ & $17,60 \pm 10,26$ & 0,032 \\
\hline
\end{tabular}

\subsection{Análisis de Regresión Lineal Múltiple}

Los suplementos de zinc se asociaron significativamente con reducción del Índice CPOS (beta = -3,9; $\mathrm{p}=0,033)$. También el cepillado dental con pasta dental se asoció con reducción del Índice de Superficies Cariadas (CPOS) (beta $=-7,14 ; \mathrm{p}=0,033)$ (Tabla 4).

Tabla 4. Regresión Lineal Múltiple: CPOS final y variables basales.

\begin{tabular}{|c|c|c|c|}
\hline Variables explicativas & Coeficiente $\beta$ & $\mathrm{EE}^{2}$ & $\mathrm{p}$ \\
\hline WAZ inicial (DE) & $-0,130$ & 1,386 & 0,925 \\
\hline HAZ inicial (DE) & $-0,269$ & 1,578 & 0,865 \\
\hline Zinc inicial ( $\mathrm{ug} \mathrm{dl^{-1 }}$ ) & $-0,001$ & 0,040 & 0,990 \\
\hline $\operatorname{IgA}$ inicial $\left(\mathrm{mg} \mathrm{dl}^{-1}\right)$ & $-0,056$ & 0,137 & 0,682 \\
\hline Cepillado dientes am & $-1,247$ & 1,705 & 0,466 \\
\hline Cepillado dientes pm & 2,303 & 1,701 & 0,177 \\
\hline Uso de pasta dental & $-7,141$ & 3,323 & 0,033 \\
\hline Uso de agua potable & $-2,953$ & 1,837 & 0,110 \\
\hline Suplemento zinc/placebo & $-3,972$ & 1,852 & 0,033 \\
\hline Sexo femenino/masculino & 1,047 & 1,711 & 0,541 \\
\hline
\end{tabular}

Los suplementos de zinc se asociaron con reducción del Índice CPOD (beta $=-1,33 ; p=0,05$ ). También el enjuague con agua potable en el cepillado de dientes se asoció con reducción del Índice de Piezas Dentarias Cariadas (CPOD) (beta $=-1,42 ; p=0,035)$ (Tabla 5).

Tabla 5. Regresión Lineal Múltiple: CPOD final y variables basales.

\begin{tabular}{lccc}
\hline Variables explicativas & Coeficiente $\beta$ & $\mathrm{EE}^{2}$ & $\mathrm{p}$ \\
\hline WAZ inicial (DE) & $-0,124$ & 0,504 & 0,806 \\
HAZ inicial (DE) & 0,422 & 0,573 & 0,463 \\
Zinc inicial ( $\mathrm{ug} \mathrm{dl}^{-1}$ ) & $-0,002$ & 0,015 & 0,910 \\
${\text { IgA inicial }\left(\mathrm{mg} \mathrm{dl}^{-1} \text { ) }\right.}_{\text {Cepillado dientes am }}^{-0,011}$ & 0,050 & 0,827 \\
\hline
\end{tabular}

\footnotetext{
${ }^{2}$ EE: error de la estimación
} 


\begin{tabular}{lccc}
\hline Cepillado dientes pm & 1,190 & 0,618 & 0,056 \\
Uso de pasta dental & $-1,824$ & 1,208 & 0,133 \\
$\begin{array}{l}\text { Uso de agua potable en el } \\
\text { enjuague }\end{array}$ & $-1,421$ & 0,668 & 0,035 \\
$\begin{array}{l}\text { Suplemento zinc/placebo } \\
\text { Sexo femenino/masculino }\end{array}$ & $-1,328$ & 0,673 & 0,050 \\
\hline
\end{tabular}

La concentración inicial de zinc se asoció negativamente con la concentración final de IgA. Es decir, por cada microgramo de zinc plasmático la concentración de IgA disminuye en 0,043 miligramos $(\mathrm{p}=0,011)$ (Tabla 6).

Tabla 6. Regresión Lineal Múltiple: Inmunoglobulina A salival final y variables basales.

\begin{tabular}{lccc}
\hline Variables explicativas & Coeficiente $\beta$ & $\mathrm{EE}^{2}$ & $\mathrm{p}$ \\
\hline WAZ inicial (DE) & $-0,964$ & 0,571 & 0,093 \\
HAZ inicial (DE) & 1,093 & 0,657 & 0,098 \\
Zinc inicial (ug dl ${ }^{-1}$ ) & $-0,043$ & 0,017 & 0,011 \\
Cepillado dientes am & 0,314 & 0,707 & 0,657 \\
Cepillado dientes pm & 0,895 & 0,699 & 0,202 \\
Uso de pasta dental & $-1,025$ & 1,374 & 0,457 \\
Uso de agua potable & $-0,104$ & 0,762 & 0,892 \\
Suplemento zinc/placebo & 0,130 & 0,766 & 0,865 \\
Sexo femenino/masculino & 0,806 & 0,706 & 0,256 \\
\hline
\end{tabular}

La talla final expresada como "score" Z (HAZ) no estuvo asociada significativamente con ninguna de las variables independientes en el modelo de Regresión Lineal Múltiple (datos no mostrados).

\section{DISCUSIÓN Y CONCLUSIONES}

\subsection{Magnitud del problema}

Pérdida de piezas dentarias y restauraciones de piezas cariadas, que se mide mediante el índice CPOD, son un grave problema de salud pública en los niños de escuelas urbano marginales de la ciudad de Cuenca. En efecto, el CPOD basal fue de 9,16, es decir, casi 3 veces más alto que el límite de 3,5 establecido para poblaciones escolares saludables, según la WHO (2000). Por las características de esta población el hallazgo puede extenderse a niños escolares de la sierra ecuatoriana. El índice también es más alto que en otros países de Latinoamérica. Así el índice de caries dental CPOD en Colombia es de 3,6 en niños de 6-9 años y de 2,5 en niños de 10-12 años (Secretaria de Salud de Bogotá, 2007), en Brasil es de 2,5 (Ariede Petinuci y col., 2005). El índice CPOS, también revela la alarmante magnitud del problema entre nuestros niños. En efecto, el valor inicial de CPOS fue de 17,09, frente al límite de 4 establecido para poblaciones saludables. Debe subrayarse que los niños de nuestro estudio tenían un estado crónico de deficiencia nutricional como sugiere su retraso de talla.

\subsection{Beneficio de suplementos de zinc}

Al concluir las 42 semanas de suplementación, los niños que recibieron zinc tuvieron menor índice de superficies cariadas, CPOS, que los niños que recibieron placebo. No hubo episodios de reacciones indeseables al jarabe. No se han referido estudios longitudinales de intervención con suplementos de zinc para evaluar su efecto en la prevalencia de caries, pero un estudio transversal reciente encontró 
que niños con deficiencia de zinc en el plasma sanguíneo $\left(<70 \mu \mathrm{g} \mathrm{dl}^{-1}\right)$ tuvieron mayor prevalencia de caries que niños sin deficiencia (Atosoy y Ulosoy, 2012). Otro estudio en niños mostró que bajas concentraciones salivales de zinc se relacionan con múltiples caries (Zahir y Sarkar, 2006). Estos investigadores discutieron probables beneficios directos del zinc en la protección de las piezas dentarias. En efecto, algunos estudios in vitro han mostrado que el zinc local protege el esmalte (Lynch, 2011) y otro estudio reciente ha mostrado que el zinc local inhibe a las metaloproteasas de la dentina que degradan el colágeno y favorecen las caries (Toledano y col., 2012). Puede asumirse que el mejoramiento sistémico del status de zinc se relaciona con mayor concentración salival de este mineral, lo que contribuiría a proteger las piezas dentarias.

El análisis de Regresión Lineal Múltiple mostró que el suplemento de zinc se asoció con 3,9 puntos menos de CPOS y 1,33 puntos menos de CPOD, cuando se controlaron algunas variables tanto nutricionales, cuanto de higiene oral y de condiciones de vida. Este hallazgo sugiere que el zinc podría estar implicado en mecanismos antibacterianos en la cavidad oral que intervienen en la contención de patógenos asociados a caries. Precisamente, un estudio reciente ha mostrado que el zinc previene el crecimiento de Streptococcus sobrinus con un $80 \%$ de reducción de las cuentas bacterianas (Berdan y Hanley, 2010).

Un hallazgo interesante en este estudio es que a medida que las concentraciones plasmáticas de zinc incrementan, las concentraciones de $\operatorname{IgA}$ tienden a disminuir. Esta asociación parece lógica puesto que si el zinc controla los patógenos orales por ende disminuye el desafío antigénico y la inducción de la respuesta inmune dependiente de anticuerpos. Es posible que el control de los patógenos orales se deba a que el zinc sistémico estimula la producción de péptidos antibacterianos en las células de la inmunidad innata de la mucosa oral. Precisamente, se ha demostrado que el zinc induce producción del péptido cathelicidine (LL-37), un potente microbicida, en células cultivadas (Taludker y col., 2011). Posiblemente otras sustancias también pueden estar implicadas.

\subsection{Otras prácticas beneficiosas}

Los análisis de regresión Lineal Múltiple mostraron que el cepillado dental con pasta y el uso de agua potable para el aseo oral, se asocian significativamente con menor CPOS y CPOD, respectivamente, lo cual muestra que estos hábitos asociados a un mejoramiento del status de zinc pueden tener un efecto significativo en la reducción de caries en estos niños escolares de talla baja. Pastas dentífricas con zinc tienen efecto antibacteriano (Hu y col., 2010), pero la administración oral de zinc puede mantener una concentración salival más constante y, además, ofrecer otros beneficios relacionados con el crecimiento y la prevención de infecciones respiratorias y entéricas.

Fortalezas de este estudio son su diseño aleatorizado y doble ciego. Entre las debilidades señalamos que no se alcanzó el tamaño estimado de los grupos y la pérdida de algunos niños antes de que se iniciara la suplementación debido a la larga duración de las mediciones basales. Así como la interrupción de la suplementación durante el período de vacaciones escolares.

\section{AGRADECIMIENTOS}

Los autores expresan sus sinceros agradecimientos al FUNDACYT (Proyecto PIC-002), la Dirección de Investigación de la Universidad de Cuenca y su Facultad de Odontología por el apoyo financiero. Además, los autores desean reconocer a la Universidad Central del Ecuador por la medición de zinc en el plasma, y la Corporación Ecuatoriana de Biotecnología, particularmente a la doctora Bertha Estrella, por su colaboración en los análisis estadísticos. Un agradecimiento especial a los estudiantes ayudantes de investigación de la Facultad de Odontología en el periodo 2005-2008. Por último, pero no menos importante, los autores expresan gratitud a los directores y profesores de las escuelas: Medardo Neira, Cornelio Crespo, Nicolás Sojos, Enriqueta Cordero y Aurelio Ochoa por apoyar el estudio y administrar los suplementos, y a las niñas y niños, que nos permitieron realizar las mediciones previstas y que aceptaron tomar los suplementos. 


\section{BIBLIOGRAFÍA}

Ariede Petinuci, P., K. Polido Kaneshiro, M.A. Rabelo, 2005. Dental caries and dental fluorosis in 712-yearold schoolchildren in Catalão, Goiás, Brazil. J. Appl. Oral. Sci., 13(1), 35-40.

Atasoy, H.B., Z.I. Ulusoy, 2012. The relationship between zinc deficiency and children's oral health. Pediatr. Dent., 34(5), 383-6.

Beck, F.W., A.S. Prasad, J. Kaplan, J.T. Fitzgerald, G.J. Brewer, 1997. Changes in cytokine production and $\mathrm{T}$ cell subpopulations in experimentally induced zinc-deficient humans. Am. J. Physiol., 272(6 Pt 1), E1002-7.

Aydin Sevinç, B., L. Hanley, 2010. Antibacterial activity of dental composites containing zinc oxide nanoparticles. J. Biomed. Mater. Res. B Appl. Biomater., 94, 22-31.

Bhutta, Z.A., R.E. Black, K.H. Brown, J.M. Gardner, S. Gore, A. Hidayat, F. Khatun, R. Martorell, N.X. Ninh, M.E. Penny, J.L. Rosado, S.K. Roy, M. Ruel, S. Sazawal, A. Shankar, 1999. Prevention of diarrhea and pneumonia by zinc supplementation in children in developing countries: pooled analysis of randomized controlled trials. J. Pediatr., 135(6), 689-97.

Brown, K.H., J.M. Peerson, J. Rivera, L.H. Allen, 2002. Effect of supplemental zinc on the growth and serum zinc concentrations of prepubertal children a meta-analysis of randomized controlled trials. Am. J. Clin. Nutr., 75(6), 1062-1071.

Cerklewski, F.L., 1981. Effect of suboptimal zinc nutrition during gestation and lactation on rat molar tooth composition and dental caries. J. Nutr., 111(10), 1780-3.

Fraker, P.J., L.E. King, T. Laakko, T.L. Vollmer, 2000. The dynamic link between the integrity of the immune system and zinc status. J. Nutr., 130, 1399S-406S.

Freire, W., 1988. Diagnostico de la situación alimentaria, nutricional y de salud de la población ecuatoriana menor de 5 años: Resumen. CONADE-M.S.P., Ecuador. Descargado de http://www.repositorio.iaen.edu.ec .../DIAGNOSTICO\%20DE\%20... en 2012, 62 págs.

Hau, J., E. Andersson, H.E. Carlsson, 2001. Development and validation of a sensitive ELISA for quantification of secretory IgA in rat saliva and faeces. Lab. Animal., 35(4), 301-6.

Hu, D., P.K. Sreenivasan, Y.P. Zhang, W. De Vizio, 2010. The effects of a zinc citrate dentifrice on bacteria found on oral surfaces. Oral Health Prev. Dent., 8(1),:47-53.

International Zinc Nutrition Consultative Group (IZiNCG), K.H. Brown, J.A. Rivera, Z. Bhutta, R.S. Gibson, J.C. King, B. Lönnerdal, M.T. Ruel, B. Sandtröm, E. Wasantwisut, C. Hotz, 2004. International Zinc Nutrition Consultative Group (IZiNCG) Technical Document \#1. Assessment of the risk of zinc deficiency in populations and options for its control. Food. Nutr. Bull., 25(1 Suppl 2), S99-203.

Johnson, D.A., O.F. Alvares, 1984. Zinc deficiency-induced changes in rat parotid salivary proteins. $J$. Nutr.,114(10), 1955-64.

Larrea, C., W. Freire, Ch. Lutter, 2001. Equidad desde el principio: Situación nutricional de los niños ecuatorianos. Organización Panamericana de la Salud, Washington D.C., EE.UU., 41 págs.

Lee, S.R., H.K. Kwon, K.B. Song, Y.H. Choi, 2004. Dental caries and salivary immunoglobulin A in Down syndrome children. J. Paediatr. Child Health., 40(9-10), 530-3.

Lynch, R.J., 2011. Zinc in the mouth, its interactions with dental enamel and possible effects on caries; a review of the literature. Int. Dent. J., 61 Suppl 3, 46-54.

Nicolau, B., W. Marcenes, P. Allison, A. Sheiham, 2005. The life course approach: explaining the association between height and dental caries in Brazilian adolescents. Community Dent. Oral Epidemiol., 33(2), 93-8.

Peres, K.G., R. Latorre Mdo, M.A. Peres, J. Traebert, M. Panizzi, 2003. Impact of dental caries and dental fluorosis on 12-year-old school children's self-perception of appearance and chewing. Cad. Saude Publica, 19(1), 323-30.

Prasad, A.S., S. Meftah, J. Abdallah, J. Kaplan, G.J. Brewer, J.F. Bach, M. Dardenne, 1988. Serum thymulin in human zinc deficiency. J. Clin. Invest., 82(4), 1002-10. 
PRECONC,1992. Odontología Preventiva (Curso 1). Programa de Educación Continua Odontológica No Convencional, Organización Panamericana de la Salud. Buenos Aires, págs. 28-32.

Proctor, G.B., G.H. Carpenter, 2001. Chewing stimulates secretion of human salivary secretory immunoglobulin A. J. Dent. Res., 80(3), 909-13.

Sazawal, S., R.E. Black, M.K. Bhan, S. Jalla, A. Sinha, N. Bhandari, 1997. Efficay of zinc supplementation in reducing the incidence and prevalence of acute diarrhea- a community-based, double-blind, controlled trial. Am. J. Clin. Nutr., 66(2), 413-418.

Secretaria de Salud de Bogotá, Grupo Salud Oral, 2007. Proyecto de Desarrollo de Autonomía de salud Oral. Bogotá DC, Colombia. Descargado de http://saludpublicabogota.org/wiki/images/3/34/Salud_oral.pdf en noviembre 2012.

Sempértegui, F., B. Estrella, E. Correa, L. Aguirre, B. Saa, M. Torres, F. Navarrete, C. Alarcón, J. Carrión, Rodríguez, J.K. Griffiths, 1996. Effects of short-term zinc supplementation on cellular immunity, respiratory symptoms, and growth of malnourished Equadorian children. Eur. J. Clin. Nutr., 50(1), 42-46.

Shankar, A.H., A.S. Prasad, 1998. Zinc and immune function : the biological basis of altered resistance to infection. Am. J. Clin. Nutr., 68(2 Suppl), 447S-463S.

Smith, P.G., R.H. Morrow, 1991. Methods for field trials of intervention against tropical diseases: a toolbox. Oxford University Press, New York, 352 págs.

Struska, A., M. Mielnik-Blaszczak, 2003. Correlation between frequency of caries in deciduous dentition and physical development of children in the first six months of life. Ann. Univ. Mariae Curie Sklodowska Med., 58(1),179-84.

Tar, I., E. Nemes, J. Nemes, M. Alberth, G. Keszthelyi, 1999. The role of salivary immunoglobulins (secretory $\operatorname{IgA}, \operatorname{IgM}, \operatorname{IgG}$ ) in caries prevalence and primary B-cell deficiency. Fogorv Sz., 92(11), 331-8.

Talukder, P., T. Satho, K. Irie, T. Sharmin, D. Hamady, Y. Nakashima, N. Kashige, F. Miake, 2011. Trace metal zinc stimulates secretion of antimicrobial peptide LL-37 from Caco-2 cells through ERK and p38 MAP kinase. Int. Immunopharmacol., 11(1), 141-4.

Toledano, M., M. Yamauti, E. Osorio, R. Osorio, 2012. Zinc-inhibited MMP-mediated collagen degradation after different dentine demineralization procedures. Caries Res., 46, 201-7.

WHO, 2000. Global Oral Health Data Bank and WHO Oral Health Country/Area Profile Programme. En: Petersen, P.E., Challenges to improvement of oral health in the 21st century - the approach of the WHO Global Oral Health Programme. Int. Dent. J., 54, 329-343.

WHO, 1987. Oral health surveys. Basic Methods ( $3^{\text {rd }}$ Ed.). World Health Organization, Geneve, Suiza.

Wuehler, S.E., F. Sempértegui, K.H. Brown, 2008. Dose-response trial of prophylactic zinc supplements, with or without copper, in young Ecuadorian children at risk of zinc deficiency. Am. J. Clin. Nutr., 87, 723-33.

Zahir, S., S. Sarkar, 2006. Study of trace elements in mixed saliva of caries free and caries active children. J. Indian Soc. Pedod. Prev. Dent., 24(1), $27-9$.

Zhou, J.R., N.M. Cnara, J.W. Erdman Jr., 1993. Bone zinc is poorly released in young, growing rats fed marginally zinc-restricted diet. J. Nutr., 123(8), 1383-8. 